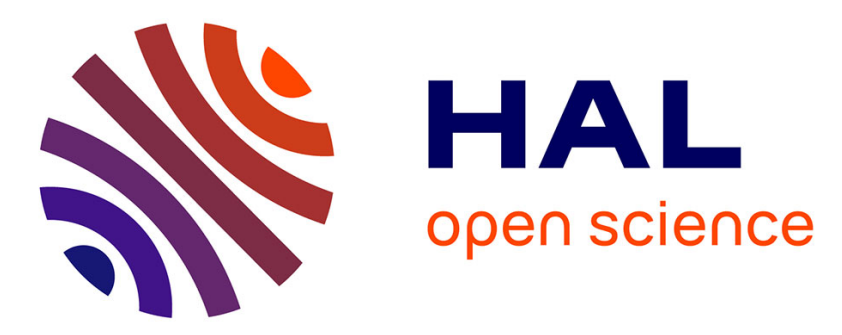

\title{
Etude de l'ordre local autour du fer dans des verres silicatés naturels et synthétiques à l'aide de la spectrométrie d'absorption $\mathrm{X}$
}

\author{
G. Calas, P. Levitz, J. Petiau, P. Bondot, G. Loupias
}

\section{- To cite this version:}

G. Calas, P. Levitz, J. Petiau, P. Bondot, G. Loupias. Etude de l'ordre local autour du fer dans des verres silicatés naturels et synthétiques à l'aide de la spectrométrie d'absorption X. Revue de Physique Appliquée, 1980, 15 (6), pp.1161-1167. 10.1051/rphysap:019800015060116100 . jpa-00244836

\section{HAL Id: jpa-00244836 https://hal.science/jpa-00244836}

Submitted on 1 Jan 1980

HAL is a multi-disciplinary open access archive for the deposit and dissemination of scientific research documents, whether they are published or not. The documents may come from teaching and research institutions in France or abroad, or from public or private research centers.
L'archive ouverte pluridisciplinaire HAL, est destinée au dépôt et à la diffusion de documents scientifiques de niveau recherche, publiés ou non, émanant des établissements d'enseignement et de recherche français ou étrangers, des laboratoires publics ou privés. 


\title{
Etude de l'ordre local autour du fer dans des verres silicatés naturels et synthétiques à l'aide de la spectrométrie d'absorption $\mathbf{X}$
}

\author{
G. Calas, P. Levitz, J. Petiau (*), P. Bondot et G. Loupias \\ Laboratoire de Minéralogie-Cristallographie, L.A., CNRS 09, 4 place Jussieu, 75230 Paris Cedex 05, France, et \\ Laboratoire pour l'Utilisation du Rayonnement électromagnétique (**), Paris-Sud, 91405 Orsay, France
}

(Reçu le 15 novembre 1979, révisé le 22 février 1980, accepté le 26 février 1980)

\begin{abstract}
Résumé. - Nous présentons les premiers résultats obtenus par spectroscopie d'absorption X sur les distances feroxygène et l'état chimique du fer dans quatre verres silicatés contenant le fer à l'état de valence II ou III. Deux ont été préparés dans des conditions d'oxydation contrôlées pour obtenir l'une ou l'autre valence; les deux autres sont des verres naturels de composition et contexte géologique différents.

Les distances Fe-O ont été déterminées par analyse des oscillations EXAFS : la distance Fe (II)-O est trouvée égale à $2,07 \AA$ et elle est inférieure aux valeurs trouvées en moyenne pour les sites hexacoordonnés dans les silicates cristallisés; la distance Fe (III)-O est de 1,91 $\AA$ et concorde assez bien avec la valeur attendue pour un site tétracoordonné de fer trivalent. Dans tous les cas, la distribution radiale des distances Fe-O semble faible.

Des informations sur la valence, la coordinance et l'ionicité de la liaison Fe-O sont déduites de l'étude fine du seuil $\mathrm{K}$ du fer et comparées à des composés cristallins.
\end{abstract}

\begin{abstract}
We present some first results obtained by X-ray absorption spectroscopy on Fe-O distances and chemical state of iron in four silicate glasses containing ferric or ferrous ions. Two of these glasses are prepared under controlled oxidation conditions in order to obtain a defined oxidation state ; the other two are natural glasses with different compositions and geological history.

Fe-O distances are determined by analyzing EXAFS oscillations : Fe (II)-O distance is found to be $2.07 \AA$, smaller than the mean values observed in crystalline silicates for hexacoordinated sites; the Fe (III)-O distance, $1.91 \AA$, fits reasonably well with the hypothesis of a tetracoordinated site of Fe (III). Radial distribution of Fe-O distances seems to be small in all glasses.

It is possible to deduce informations on oxidation state, coordinence and ionicity of the $\mathrm{Fe}-\mathrm{O}$ bond from $\mathrm{Fe}-\mathrm{K}$ absorption edge and a comparison with crystallized compounds is made.
\end{abstract}

1. Introduction. - Le problème posé par l'étude spectroscopique des éléments de transition dans les verres multicomposants, tels les verres silicatés, est dû au fait que l'on sait mal séparer, généralement, les effets purement géométriques (symétrie locale, distances métal-oxygène) des effets chimiques (nature plus ou moins covalente des liaisons métal-oxygène, charges réelles des ligandes [1]). La spectrométrie d'absorption $\mathrm{X}$ permet, contrairement aux autres techniques, d'obtenir des informations géométriques [2] autour de l'élément étudié, ce qui peut servir à re-discuter des données classiques, tels les spectres d'absorption optique ou les spectres Mössbauer dans le cas du fer.

(*) Et E.N.S.J.F. Paris.

${ }^{(* *)}$ Laboratoire propre du C.N.R.S., conventionné à l'université de Paris-Sud.
La spectrométrie d'absorption $\mathrm{X}$ à partir du seuil d'un élément contient schématiquement deux zones et deux types d'information :

- La structure même du seuil (sur environ $30 \mathrm{eV}$ ) et sa position en énergie sont déterminées par l'état électronique de l'élément dans le matériau. L'effet principal est le déplacement chimique lié à la valence de l'élément considéré ; cependant, la coordinance et le caractère plus ou moins ionique ou covalent de la liaison ont aussi une influence sur la position et la structure du seuil.

- Les oscillations (EXAFS) du spectre étendu à quelques centaines d'eV au-dessus du seuil contiennent des informations géométriques (distances interatomiques, coordinance).

Signalons dès maintenant deux caractéristiques essentielles communes à tous les verres que nous 
avons étudiés et qui ont eu une grande importance pour le développement de notre étude de l'EXAFS :

- Seule la première couche d'oxygène entourant l'atome de fer participe au spectre EXAFS, alors que les spectres de composés cristallisés contiennent les contributions de plusieurs couches successives (la distribution des distances atomiques sur les autres couches: dépasse donc dans les verres la limite 0,2$0,3 \AA$ au-delà de laquelle l'effet d'interférences qui crée les oscillations EXAFS est pratiquement brouillé).

- La distribution des distances $\mathrm{Fe}-\mathrm{O}$ sur la première couche semble au contraire particulièrement faible dans les verres. Une constatation semblable a été faite dans les quelques travaux déjà publiés relatifs à l'utilisation de l'EXAFS dans des composés amorphes [3]. Il faut insister sur ce fait, puisqu'il constitue la condition par laquelle l'EXAFS se révèle un outil adapté à l'étude de l'ordre local dans ces verres.

Nous n'avons pas pu, à partir des mesures faites sur ces verres, interpréter systématiquement l'amplitude absolue d'où la coordinance ou en tout cas le nombre de voisins contribuant à la structure EXAFS. Nous reviendrons sur cette limitation dans nos conclusions.

2. Conditions expérimentales. - 2.1 EchanTILLONS ÉTUDIÉs. - Quatre verres ont été étudiés :

- Un verre de composition $\mathrm{Na}_{2} \mathrm{Si}_{2} \mathrm{O}_{5}$ dopé à $6 \% \mathrm{Fe}_{2} \mathrm{O}_{3}$ et fondu à $1100^{\circ} \mathrm{C}$ à l'air libre pendant $3 \mathrm{~h}$; la trempe a été faite dans l'air. Il contient essentiellement du fer (III);

- Un verre à composition de pyroxène (augite) : $\mathrm{Ca}_{0,8} \mathrm{Mg}_{0,8} \mathrm{Fe}_{0,4} \mathrm{Si}_{2} \mathrm{O}_{6}$, synthétisé à $1400^{\circ} \mathrm{C}$ en atmosphère réductrice $\left(10^{-10} \mathrm{~atm}\right.$. d'oxygène) é trempé par chute dans l'eau. Il ne contient que du fer (II), l'absence de fer trivalent étant vérifiée par R.P.E.;

- Un verre basaltique de l'Erta'Ale (Afar, Ethiopie) de composition (exprimée en pourcentage poids); 49,83 $\mathrm{SiO}_{2}, 2,36 \mathrm{TiO}_{2}, 13,92 \mathrm{Al}_{2} \mathrm{O}_{3}, 2,01 \mathrm{Fe}_{2} \mathrm{O}_{3}$, 9,31 $\mathrm{FeO}, 0,19 \mathrm{MnO}, 6,38 \mathrm{MgO}, 11,33 \mathrm{CaO}$, 2,88 $\mathrm{Na}_{2} \mathrm{O}, 0,64 \mathrm{~K}_{2} \mathrm{O}$. La spectroscopie Mössbauer indique que $80 \%$ du fer est sous la forme divalente ;

- Un verre très siliceux (pantellerite) de la série de Boïna (Afar, Ethiopie) de composition : $68,43 \mathrm{SiO}_{2}$, $0,26 \mathrm{TiO}_{2}, 11,10 \mathrm{Al}_{2} \mathrm{O}_{3}, 3,51 \quad \mathrm{Fe}_{2} \mathrm{O}_{3}, 3,59 \mathrm{FeO}$, $0,19 \mathrm{MnO},<0,01 \mathrm{MgO}, 0,56 \mathrm{CaO}, 6,0 \quad \mathrm{Na}_{2} \mathrm{O}$, $4,5 \mathrm{~K}_{2} \mathrm{O}$. Ce verre contient $75 \%$ du fer à l'état divalent, ce qui indique une grande différence avec l'analyse chimique par voie humide.

Ces verres ont été comparés à quelques références cristallisées, mesurées dans les mêmes conditions.

2.2 CHOIX DES RÉFÉRENCES. - Nous avons utilisé trois références : $\mathrm{Fe}_{x} \mathrm{O}(x=0,91), \mathrm{Fe}_{2} \mathrm{SiO}_{4}$ (fayalite) et $\mathrm{Fe}_{2} \mathrm{O}_{3}$. Dans $\mathrm{FeO}$, le fer (II) se trouve en site octaédrique avec $d(\mathrm{Fe}-\mathrm{O})=2,15 \AA$. Le cas de $\mathrm{Fe}_{2} \mathrm{SiO}_{4}$ est plus complexe car le fer (II) occupe deux sites octaédriques différents, notés $M 1$ et $M 2$; le premier est très peu déformé avec $d(\mathrm{Fe}-\mathrm{O})=2,16 \AA$ et le second moins régulier avec $d(\mathrm{Fe}-\mathrm{O})=2,18 \AA$ en moyenne. La distribution des distances dans ce dernier site est environ $0,21 \AA$. Dans $\mathrm{Fe}_{2} \mathrm{O}_{3}$, le fer (III) octaédrique est entouré de trois oxygènes à $1,91 \AA$ et de trois autres à $2,06 \AA$, donnant une distribution de $0,15 \AA$ autour de la valeur moyenne $1,985 \AA$. Un quatrième échantillon a été choisi en tant que cas extrême pour voir l'effet d'une distribution importante des distance $\mathrm{Fe}-\mathrm{O}$; il s'agit d'une augite volcanique caractérisée par deux sites très différents : l'un (M1) est un octaèdre semblable au site $\mathrm{M} 2$ de la fayalite avec $d(\mathrm{Fe}-\mathrm{O})=2,15 \AA$, et l'autre (M2) est très déformé, le fer étant entouré de six oxygènes à des distances fortement distribuées $(\sigma>0,45 \AA)$; la moyenne des distances $\mathrm{Fe}-\mathrm{O}$ dans les deux sites est de $2,22 \AA$.

2.3 Mesure Des SPECTRES D'ABSORPTION X. - Pour la compréhension du phénomène et les détails sur la réalisation des expériences, nous renvoyons à l'article général de Raoux et al. [2].

Les mesures ont été réalisées sur le rayonnement synchrotron fourni par l'anneau DCI à Orsay; les spectres sont faits au seuil $\mathrm{K}$ du fer $\left(E_{\mathrm{K}} \sim 7111 \mathrm{eV}\right)$. Le monochromateur utilisé est un monocristal channel-cut en silicium utilisant la réflexion 220 ; sa fonction de résolution globale à $7 \mathrm{keV}$ a une largeur de $1,5 \mathrm{eV}$ et permet de mesurer des déplacements de seuil avec une précision de 0,2-0,3 eV. L'absorption par l'échantillon est déduite des mesures de l'intensité du faisceau monochromatique dans des chambres à ionisation placées avant et après l'échantillon, pour des orientations successives du monochromateur. Le pas, constant en énergie, est de $1 \mathrm{eV}$ environ entre deux mesures pour l'enregistrement de la structure EXAFS ef de $0,25 \mathrm{eV}$ environ pour l'étude fine des seuils.

Tous les échantillons ont été réalisés par simple dépôt de poudre entre deux bandes de ruban adhésif; les mesures rapportées ici ont été effectuées à la température ambiante. Comme les éléments constituants de ces verres sont légers ( $\mathrm{Si}, \mathrm{Al}, \mathrm{O} \ldots)$, les spectres sont mesurables par absorption avec une bonne précision jusqu'à des teneurs en fer de $1 \%$. La précision des mesures des amplitudes d'oscillations est limitée par la texture, inévitablement inhomogène, des échantillons et la contamination du faisceau par du rayonnement d'énergie double dû à la réflexion harmonique 440 sur le cristal monochromateur (quelques $\%$ ).

L'acquisition des données (intensités avant et après l'échantillon en fonction de l'énergie) et le pilotage du monochromateur sont effectués à l'aide d'un miniordinateur PDP 11.04, dans un montage mis au point par P. Lagarde et D. Raoux. Les spectres EXAFS ont été mesurés de 6800 à $7500 \mathrm{eV}$, la limitation à $7500 \mathrm{eV}$ étant imposée par la présence à cette énergie d'une perturbation brutale de l'intensité du faisceau monochromatisé (glitch dû à la coexistence, pour cette 
position du monochromateur, de la réflexion (220) et d'autres réflexions importantes).

2.4 ANAL YSE DES SPECTRES EXAFS. - Les diverses étapes du traitement sont présentées ici brièvement. La modulation EXAFS $\chi(E)$ est extraite de l'absorption globale

$$
\mu(E)=\log \frac{I_{0}}{I}
$$

en soustrayant à $\mu(E)$ un polynôme de degré ajustable et en normalisant les oscillations par rapport à l'absorption totale des électrons K (c'est-à-dire l'amplitude du seuil). On obtient un spectre représenté sur les figures $1 a$ et $2 a$ dans le cas de $\mathrm{Fe}_{2} \mathrm{SiO}_{4}$ (fayalite) et du verre à composition d'augite.

Un filtrage par double transformation de Fourier est effectué pour sélectionner la contribution $\chi(k)$ relative à la première couche de voisins :

- la première transformation donne dans l'espace réel la distribution radiale centrée sur l'atome de fer (courbes $1 b$ et $2 b$ );

- la transformation du pic de l'espace réel attaché
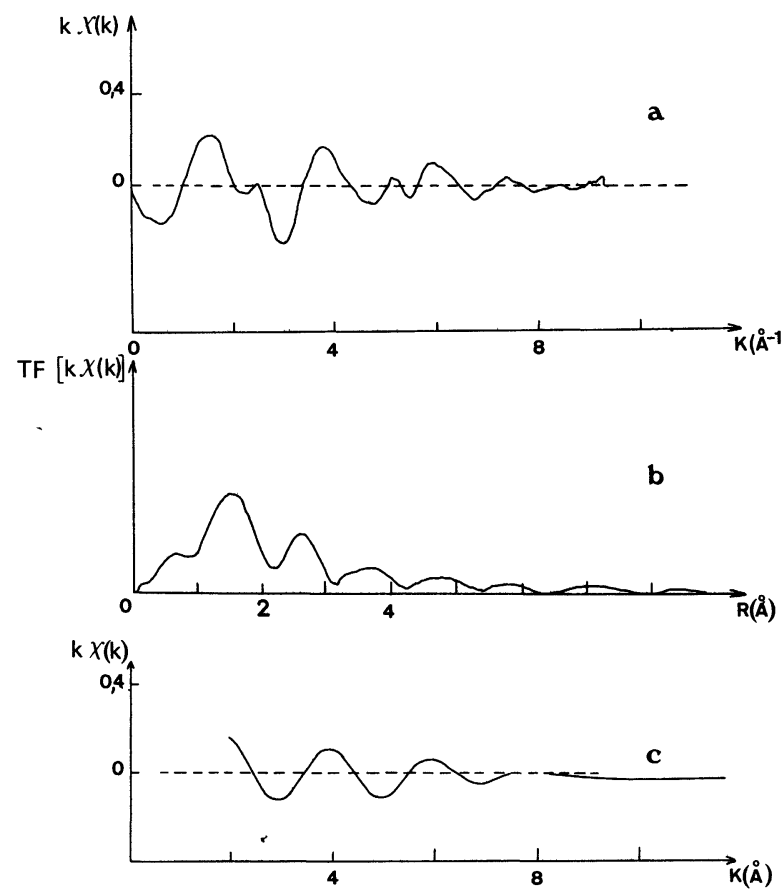

Fig. 1. - Etape de l'analyse du spectre EXAFS de $\mathrm{Fe}_{2} \mathrm{SiO}_{4}$ (fayalite) :a) partie oscillante $\chi(k)$ extraite de l'absorption globale, b) fonction de distribution radiale obtenue par transformation de Fourier de $\chi(k), c)$ transformée de Fourier inverse du pic correspondant à la couche $\mathrm{Fe}-\mathrm{O}$ (la fenêtre est indiquée sur la Fig. b).

[EXAFS spectrum analysis in fayalite $\left.\left(\mathrm{Fe}_{2} \mathrm{SiO}_{4}\right): a\right)$ oscillatory part $\chi(k)$ as extracted from global absorption, $b$ ) radial distribution function obtained by Fourier transform of $\chi(k), c)$ inverse Fourier transform of the peak corresponding to the first shell $\mathrm{Fe}-\mathrm{O}$ (the window is indicated on Fig. $b$ ) and indication of the minima and maxima of damped sinusoid $\sin (2 k R+\varphi(k)) \cdot]$

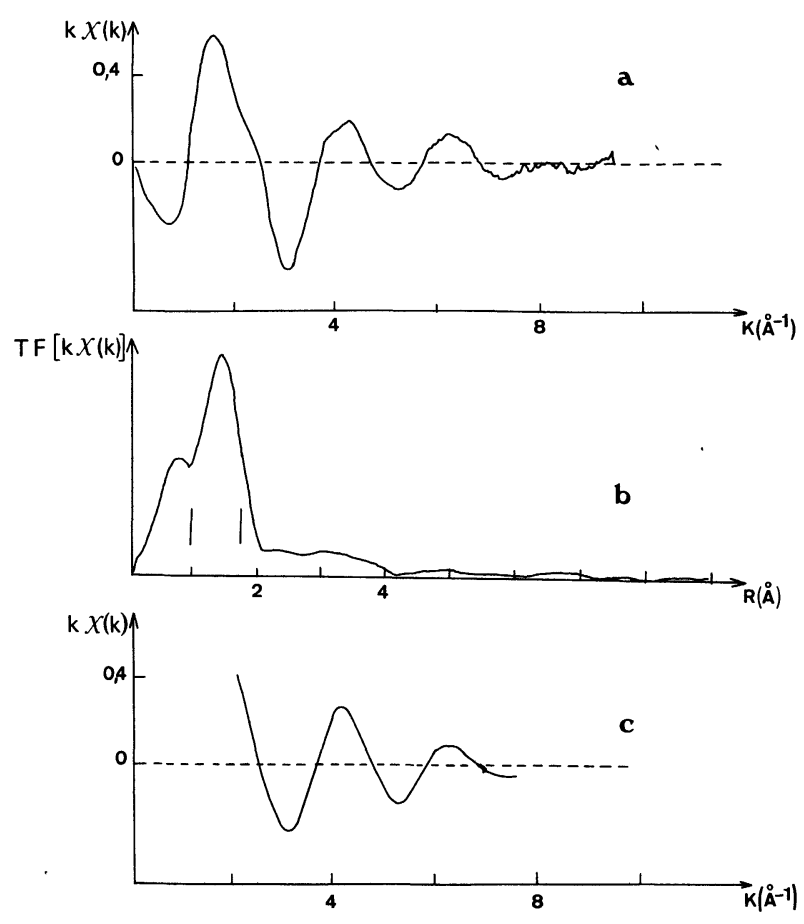

Fig. 2. - Analyse du spectre EXAFS du verre à composition d'augite. Les trois étapes $a, b, c$ sont les mêmes que sur la figure 1 . On remarque en $b$ que seule la première couche a une contribution importante.

[EXAFS spectrum analysis of an augitic glass (glass $\mathbf{n}^{\circ} 2$ ). The three steps $a, b, c$, are the same as on figure 1 . It is seen from $b$ that only one shell has a great contribution and $\mathrm{Fe}-\mathrm{O}$ distance is calculated using in $\sin (2 k R+\varphi(k))$ the $\varphi(k)$ value determined for fayalite.]

à la première couche de coordination donne une structure EXAFS partielle (courbes $1 c$ et $2 c$ ) de type sinus amorti, $\chi(k) \sim \sin [2 k R+\varphi(k)]$. L'argument dépend de la distance $R$ relative à cette couche et d'un terme de déphasage lié aux processus de diffusion par l'atome central et de rétrodiffusion par les atomes voisins [2]. $R$ est en première approximation une distance moyenne, mais l'écriture sous cette forme suppose que la distribution radiale ne soit pas trop large $(\Delta R \lesssim 0,2 \AA)$ [4]. La phase $\varphi(k)$ est déterminée à partir de composés cristallisés où les distances $\mathrm{Fe}-\mathrm{O}$ sont connues et dans lesquels l'état électronique du fer est très voisin de ce qu'il est dans les verres. Cette phase est ensuite utilisée pour calculer la distance $R$ dans les verres, en s'appuyant sur sa transférabilité du composé référence au verre.

La comparaison des seuils de la référence et du verre permet pratiquement d'éliminer les conséquences de la non-connaissance a priori du zéro de l'énergie cinétique des photoélectrons. Si les deux composés sont dans des états chimiques très voisins (même coordinance, même électrovalence), les seuils ont des positions en énergie et des structures très semblables. La figure 3 montre ainsi que pour $\mathrm{FeO}$ et le verre d'augite, les points d'inflexion coïncident à $1 \mathrm{eV}$ près. On peut alors choisir la valeur de l'énergie 


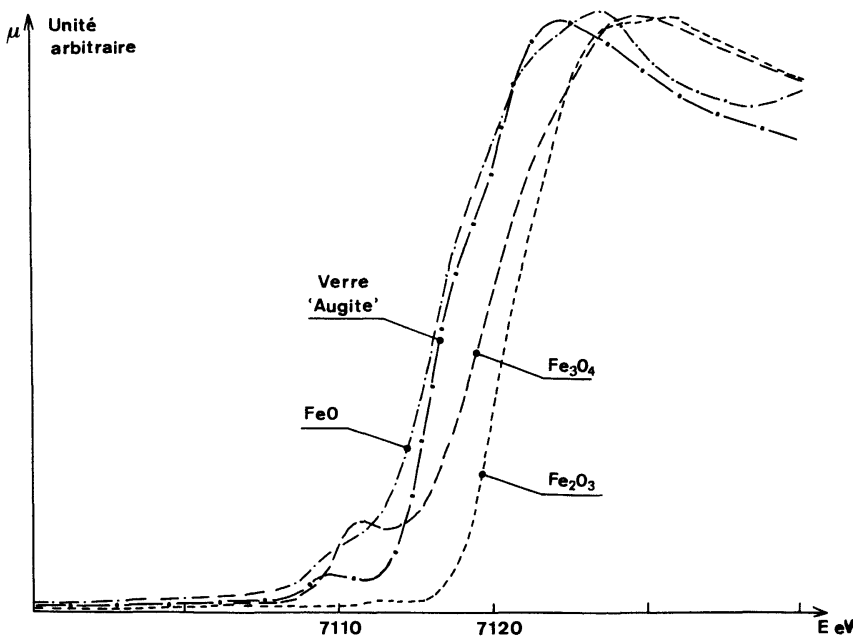

Fig. 3. - Position et structure des seuils d'absorption $K$ de trois oxydes de fer cristallisés et du verre à composition d'augite qui ne contient que du fer (II). Le lien entre la position du seuil et l'état d'oxydation du fer est clairement mis en évidence $(\Delta E \sim 5 \mathrm{eV}$ entre $\mathrm{Fe}$ (II) et $\mathrm{Fe}$ (III)).

[ $K$-absorption edge position and structure of three crystallized iron oxides and one augitic glass (containing iron (II)). The relation between edge position and valence state of iron is clearly shown $(\Delta E \sim 5 \mathrm{eV}$ between $\mathrm{Fe}$ (II) and Fe (III)).]

$E_{0}$ à des positions correspondantes par rapport aux deux seuils. Les déterminations de $R$ (verre)- $R$ (référence) restent stables à $0,01 \AA$ près lorsque la valeur de $E_{0}$ est décalée de 0 à $10 \mathrm{eV}$ de façon cohérente sur les deux composés. Cette cohérence peut se vérifier par le tracé de $R(k)$ qui donne aussi des variations de $R$ inférieures à $0,01 \AA$.

3. Résultats. - 3.1 Structure et position des SEUiLs. - Ces deux paramètres dépendent de l'état chimique de l'élément dans le composé étudié et principalement de trois facteurs : coordinance, degré d'oxydation et covalence des liaisons métal-ligande. Ce sont les deux premiers qui sont les plus importants car, dans les références utilisées pour l'étude des verres silicatés, le fer est généralement entouré d'oxygènes. L'évolution des spectres est ainsi plus complexe que celle résultant d'une simple substitution de ligandes.

3.1.1 Influence du degré d'oxydation. - Sur la figure 3 sont reportés les seuils de trois oxydes de fer et du verre d'augite (qui ne contient que du fer divalent). On constate un déplacement sensible du seuil vers les énergies élevées lorsqu'on passe du fer (II) au fer (III) : l'écart entre $\mathrm{FeO}$ et $\mathrm{Fe}_{2} \mathrm{O}_{3}$ est de 5,5 eV, donc parfaitement mesurable avec le pas de $0,25 \mathrm{eV}$ choisi. La magnétite se situe entre ces deux oxydes, mais on doit noter qu'il ne s'agit pas d'une simple translation : le maximum d'absorption coïncide avec celui de $\mathrm{Fe}_{2} \mathrm{O}_{3}$, alors que le début du seuil est plus proche de $\mathrm{FeO}$. Ceci montre bien la difficulté de mesurer les mélanges d'ions dans des états d'oxydation différents, sans une bonne connaissance des phénomènes physiques au seuil d'absorption.
On peut noter que le seuil du verre d'augite est pratiquement superposé à celui de $\mathrm{FeO}$, ce qui semble indiquer que le fer se trouve dans le même état chimique dans les deux cas.

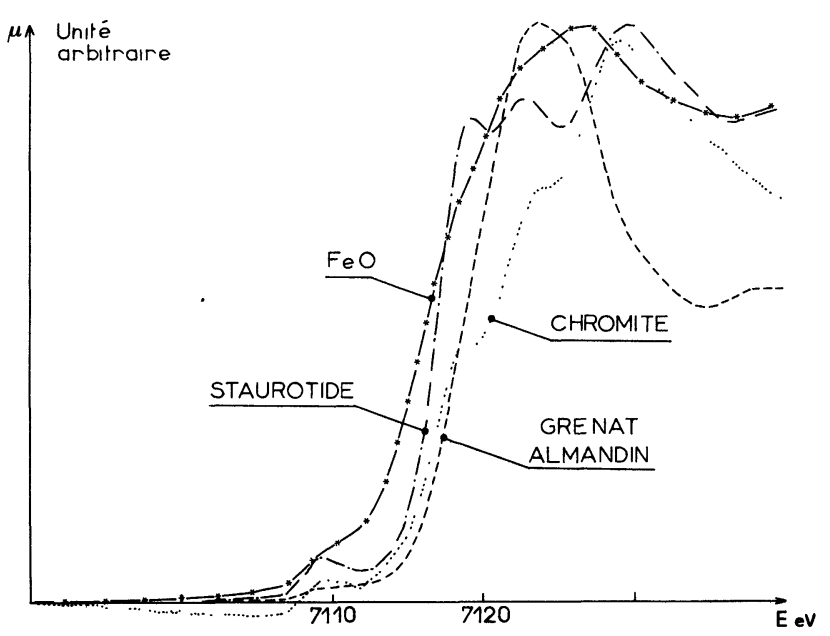

Fig. 4. - Variation du seuil d'absorption avec la coordinance du fer (II). Ces seuils correspondent à trois types de coordinance : octaédrique $\left(\mathrm{FeO}\right.$ ), tétraédrique (chromite : $\mathrm{FeCr}_{2} \mathrm{O}_{4}$ et staurotide : $\mathrm{Fe}_{2} \mathrm{Al}_{9} \mathrm{Si}_{4} \mathrm{O}_{22}(\mathrm{OH})_{2}$ ) et dodécaédrale (grenat almandin $\mathrm{Fe}_{3} \mathrm{Al}_{2} \mathrm{Si}_{3} \mathrm{O}_{12}$ ).

[Variation of $K$-absorption edge with iron coordinence, according to three types of coordinence : octahedral $(\mathrm{FeO})$, tetrahedral (chromite : $\mathrm{FeCr}_{2} \mathrm{O}_{4}$ and staurotide : $\mathrm{Fe}_{2} \mathrm{Al}_{9} \mathrm{Si}_{4} \mathrm{O}_{22}(\mathrm{OH})_{2}$ ) and dodecahedral (almandine garnet : $\mathrm{Fe}_{3} \mathrm{Al}_{2} \mathrm{Si}_{3} \mathrm{O}_{12}$ ).]

3.1.2 Influence de la coordinance. - Sur la figure 4 sont reportés les seuils de composés du fer divalent en coordinance tétraédrique (chromite, staurotide) ou dodécaédrale (grenat almandin) qui sont comparés au seuil de $\mathrm{FeO}$ (coordinance octaédrique). On constate que la position du seuil varie peu entre une coordinance 4 et une coordinance 8 , ce qui semble cohérent avec ce qui a été vu plus haut sur la relation entre l'état d'oxydation du fer et la coordinance. La structure du seuil au contraire est nettement différente dans les trois coordinances; on observe en particulier dans le cas du fer (II) tétraédrique, en plus du pic situé à faible énergie, deux pics très nets superposés à la montée du seuil d'absorption. Ils se trouvent à la même place dans les deux composés de Fe (II) tétraédrique, alors qu'ils n'existent pas dans le cas du grenat almandin.

3.1.3 Effet de la covalence. - La covalence des liaisons fer-oxygène varie généralement peu, ce qui explique la superposition des seuils d'absorption lorsque le métal se trouve dans le même état d'oxydation et la même coordinance. Lorsque la nature du ligande varie, on note au contraire un déplacement systématique de ces seuils d'absorption [5]. Nous avons comparé le seuil de $\mathrm{FeO}$ à celui de la pyrite $\mathrm{FeS}_{2}$ où le fer (II) bas spin est lié de façon fortement covalente au soufre (Fig. 5). Quoique le fer soit en coordinance octaédrique, la structure du seuil est très 


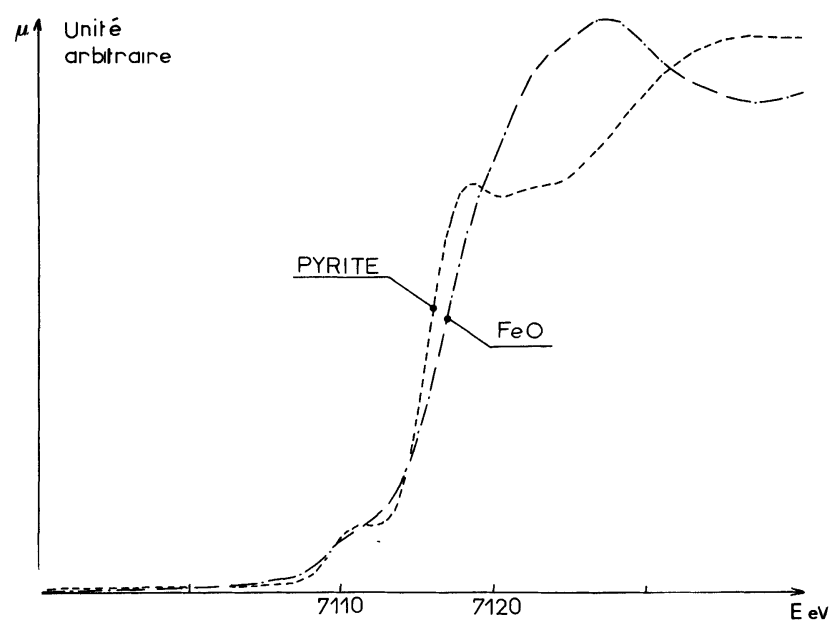

Fig. 5. - Variation de la structure du seuil en fonction de la covalence fer-ligande dans $\mathrm{FeO}$ et $\mathrm{FeS}_{2}$ (pyrite).

[Variation of $K$-absorption edge structure as a function of ironligand covalency in $\mathrm{FeO}$ and $\mathrm{FeS}_{2}$ (pyrite).]

semblable à celle des seuils caractérisant un site tétraédrique.

3.2 SPECTRES EXAFS. - 3.2.1 Limitation à la première couche de coordination. - Les spectres EXAFS des verres étudiés se caractérisent par une allure de sinusoïde amortie (Fig. 2a), alors que dans le cas des références cristallisées, ils sont beaucoup plus complexes, traduisant des interférences multiples (Fig. 1a). La transformation de Fourier de cette courbe permet d'obtenir la distribution radiale dans l'espace direct : dans les verres seule la première couche est visible (Fig. 2b), alors que l'on voit dans le cas d'un cristal comme $\mathrm{Fe}_{2} \mathrm{SiO}_{4}$ plusieurs pics correspondant à des couches différentes (Fig. 1b). L'EXAFS n'est donc sensible dans les verres qu'aux premiers voisins : les couches situées au-delà de la première couche de coordination sont trop distribuées par rapport à l'atome central (fer), ce qui provoque une destruction des interférences provenant de la rétrodiffusion par les différents atomes. Cette distribution peut être due à une variation des orientations relatives des divers polyèdres de coordination, tétraèdres $\mathrm{SiO}_{4}$ en particulier, ce qui n'est pas incompatible avec des distances bien définies à l'intérieur de ces polyèdres.

3.2.2 Distribution radiale de la première couche de coordination. - On peut estimer qualitativement la distribution radiale de la couche de premiers voisins par la largeur du pic (de forme gaussienne) correspondant dans l'espace réel. Cette largeur a deux causes principales : d'une part l'amortissement du spectre EXAFS lui-même, dû aux désordres statique et dynamique de la couche et au faible facteur de rétrodiffusion de l'oxygène, et d'autre part les apodisations introduites dans le calcul des transformées de Fourier. Ce second effet est prépondérant mais donne des largeurs égales dans tous les composés si les conditions de traitement sont identiques. Nous avons mesuré les largeurs de ce pic en symétrisant ce dernier par rapport à son flanc droit; le pic est en effet élargi sur le côté gauche (Figs. $1 b$ et $2 b$ ) par un épaulement dû à une oscillation parasite probablement en relation avec la difficulté d'ajuster correctement la fonction $\mu(E)$ (vue en 2.4) avec un polynôme simple. Dans le cas des verres nous avons obtenu des largeurs très voisines de celles mesurées dans les composés où les distances $\mathrm{Fe}-\mathrm{O}$ sont peu distribuées (comme $\mathrm{FeO})$ : la largeur $\sigma$ de la gausienne est environ de $0,53 \AA$. On observe donc que les distances $\mathrm{Fe}-\mathrm{O}$ ont une faible distribution radiale dans les verres silicatés : Hayes [3] a abouti à des conclusions semblables sur l'ordre local dans d'autres composés amorphes. Nous avons testé l'effet d'une distribution importante des distances fer-oxygène dans le cas extrême d'un pyroxène (augite : voir 2.2); l'élargissement du pic $\mathrm{Fe}-\mathrm{O}$ est très net puisque la largeur $\sigma$ est de $0,69 \AA$, ce qui montre bien que les distributions radiales importantes sont mises en évidence sans ambiguïté par la largeur du pic correspondant dans l'espace réel.

3.2.3 Transférabilité des déphasages. - Le tableau I montre que les distances fer-oxygène obtenues en transférant les déphasages entre $\mathrm{FeO}$ et $\mathrm{Fe}_{2} \mathrm{SiO}_{4}$ sont bien les distances réelles à $0,01 \AA$ près. Il est donc possible d'utiliser la transférabilité du déphasage lorsque les ions se trouvent dans des états chimiques voisins. Au contraire, l'utilisation de $\mathrm{Fe}_{2} \mathrm{O}_{3}$ comme référence conduit dans ces deux composés à des valeurs fausses, ce qui montre l'influence du degré d'oxydation. Il semble que le transfert du déphasage d'un fer

Tableau I. - Distances Fe-O déterminées par analyse de l'EXAFS dans quatre verres.

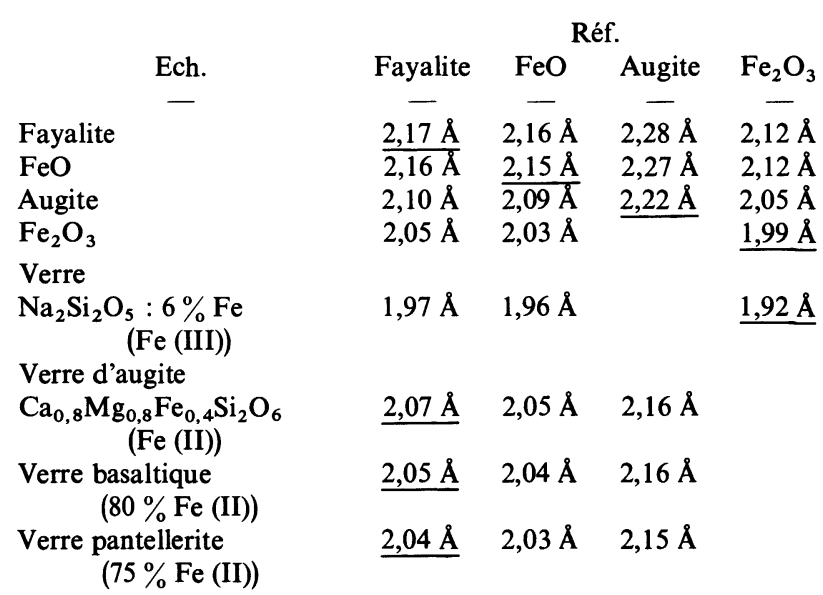

Les quatre premières lignes sont relatives aux composés cristallisés utilisés comme référence (les distances reportées montrent la bonne cohérence entre $\mathrm{FeO}$ et $\mathrm{Fe}_{2} \mathrm{SiO}_{4}$, contrairement à l'augite où les distances $\mathrm{Fe}-\mathrm{O}$ sont très distribuées).

Les distances déterminées dans les verres sont soulignées lorsque la référence est adaptée à l'état d'oxydation $\left(\mathrm{Fe}_{2} \mathrm{O}_{3}\right.$ dans le verre $\mathrm{Na}_{2} \mathrm{Si}_{2} \mathrm{O}_{5}$ " oxydé » et $\mathrm{Fe}_{2} \mathrm{SiO}_{4}$ dans le verre d'augite réduit). Dans les verres naturels on a seulement une distance $\mathrm{Fe}-\mathrm{O}$ moyenne à cause de la coexistence des deux valences du fer, d'où les valeurs intermédiaires des distances mesurées par EXAFS. 
trivalent à un fer divalent conduit à des valeurs des distances $\mathrm{Fe}-\mathrm{O}$ nettement trop petites. Nous avons choisi d'utiliser $\mathrm{Fe}_{2} \mathrm{O}_{3}$ comme référence dans le cas du verre oxydé $\mathrm{Na}_{2} \mathrm{Si}_{2} \mathrm{O}_{5}$ et $\mathrm{Fe}_{2} \mathrm{SiO}_{4}$ dans le cas des autres verres qui contiennent principalement $\mathrm{Fe}$ (II).

3.2.4 Distances obtenues dans les verres. - Les valeurs que nous avons mesurées sont reportées sur le tableau I. Ainsi que nous l'avons souligné plus haut, la faible largeur du pic correspondant à la paire $\mathrm{Fe}-\mathrm{O}$ dans l'espace direct montre que les distances correspondantes sont peu distribuées et probablement très voisines de la distance mesurée par EXAFS. Il semble en effet difficile d'admettre dans le cas d'un pic $\mathrm{Fe}-\mathrm{O}$ étroit et unique que tous les ligandes ne sont pas vus par le fer, car on observerait alors d'autres pics nettement séparés. Dans le cas de l'augite cristallisée, on trouve en revanche une distance $\mathrm{Fe}-\mathrm{O}$ nettement inférieure à la distance réelle. La distance mesurée n'est donc pas fiable lorsqu'elle est associée à une distribution radiale importante, ce qui est effectivement le cas de l'augite (voir 2.4). C'est au contraire la faible distribution observée dans les verres qui fait penser que les distances mesurées ont une signification physique correcte.

4. Discussion des résultats. - 4.1 LE FER TRIVALENT. - Le comportement du fer dans le verre oxydé $\mathrm{Na}_{2} \mathrm{Si}_{2} \mathrm{O}_{5}$ est bien connu depuis les travaux de Kurkjian et Sigety [6] qui ont montré par spectroscopie optique et effet Mössbauer que le fer (III) se trouve en site tétraédrique. La distance mesurée à partir de la référence $\mathrm{Fe}_{2} \mathrm{O}_{3}$ concorde bien avec ces résultats et la valeur de $1,91 \AA$ est très voisine de celle trouvée par G. Brown et al. [7] sur des verres à composition d'acmite $\left(\mathrm{NaFeSi}_{2} \mathrm{O}_{6}\right)$ et de feldspath-Fe $\left(\mathrm{NaFeSi}_{3} \mathrm{O}_{8}\right)$. La distance moyenne mesurée dans les verres est très voisine de ce qu'elle est dans les cristaux, l'environnement local du fer (III) ne semblant donc pas très sensible au passage du cristal au verre.

4. 2 LE FER DIVALENT. - Le fer (II) est beaucoup moins connu que le fer (III) dans les verres, car il est relativement plus difficile de synthétiser des verres ne contenant pas de fer trivalent. De nombreux problèmes résident encore dans l'interprétation des spectres optiques et Mössbauer. La spectrométrie d'absorption X apporte deux types d'informations : d'une part, le seuil d'absorption est superposable à celui d'un fer (II) octaédrique et d'autre part les distances $\mathrm{Fe}-\mathrm{O}$ sont à la fois peu distribuées et plus faibles que dans les cristaux. Le premier point indique que la coordinance du fer (II) est essentiellement octaédrique. Ces octaèdres sont plus petits que dans les composés cristallisés du fer divalent, puisque la distance Fe-O n'est que de $2,07 \AA$ dans le verre étudié, alors qu'elle est de $2,15 \AA$ dans $\mathrm{FeO}$ et de 2,16 et $2,18 \AA$ dans les deux sites de $\mathrm{Fe}_{2} \mathrm{SiO}_{4}$. Cette distance est plus faible également que dans l'aquo-complexe $(2,14 \AA)$ mais on connaît des sites, tels le site M1 des pyroxènes, où cette valeur est aussi petite; dans ces derniers, le fer (II) est cependant généralement stabilisé dans les sites M2 plus déformés.

Si l'on compare ces résultats à ceux déduits des spectres Mössbauer du fer (II) dans les verres [8, 9], on peut expliquer les faibles déplacements isomériques apparents observés $(1 \mathrm{~mm} / \mathrm{s}$ au lieu de $1,15 \mathrm{~mm} / \mathrm{s})$ par ces distances $\mathrm{Fe}-\mathrm{O}$ petites. L'asymétrie caractéristique de ces spectres [10] ne peut être interprétée que par l'existence d'au moins deux sites différents distribués. Cette distribution n'est pas radiale ainsi qu'on l'a vu plus haut, aussi faut-il invoquer plutôt une distribution de symétrie locale, ou encore une variation dans la proportion d'oxygènes très polarisés (liés au silicium), entre les différents sites occupés par le fer. L'existence de sites tétraédriques peut être également responsable des faibles déplacements isomériques apparents, mais n'étant pas visibles sur les seuils, ils sont probablement en faible proportion.

Les spectres optiques du fer (II) dans les verres sont délicats à interpréter; ils consistent en deux bandes d'absorption larges, situées dans le proche infrarouge vers 1000 et $1800 \mathrm{~nm}$. Une interprétation de ces bandes a conduit certains auteurs [11, 12] à les attribuer aux deux niveaux provenant de la décomposition du doublet ${ }^{5} \mathrm{E}_{\mathrm{g}}$, caractérisant ainsi un octaèdre déformé. Si l'on se sert des données de Faye [13], on aboutit à une estimation des distances $\mathrm{Fe}-\mathrm{O}$ nettement supérieures à $2,15 \AA$, ce qui est contredit par les données de l'EXAFS. Une autre hypothèse consiste à attribuer la bande à $1000 \mathrm{~nm}$ à un site octaédrique et la bande à $1800 \mathrm{~nm}$ à un site tétraédrique $[9,14]$. Une décomposition du spectre en gaussiennes (Fig. 6)

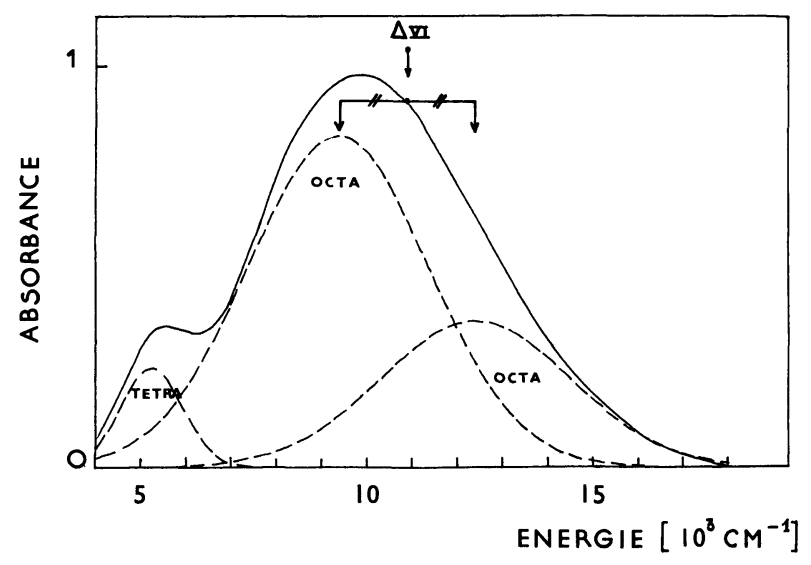

montre que la première bande peut être décomposée en réalité en deux composantes de largeur à peu près égale alors que la seconde a une forme gaussienne beaucoup plus étroite, ce qui indique que ces deux bandes ont probablement une origine différente. L'existence de sites tétraédriques est possible, ainsi qu'on l'a vu dans l'interprétation des spectres Mössbauer, mais ces sites doivent être en faible quantité car on ne les voit pas sur les seuils d'absorption. Il faut d'autre part tenir compte de la faible distribu- 
tion des distances Fe-O, si l'on admet une coexistence de sites différents. La comparaison avec les silicates cristallisés montre que dans le verre la distance $\mathrm{Fe}-\mathrm{O}$ doit être de $2,09 \AA$ pour le site octaédrique et $2 \AA$ pour le site tétraédrique, en se basant sur les bandes d'absorption optique : la différence de distances est donc relativement faible. L'information moyenne fournie par l'EXAFS ne permet pas de lever cette incertitude, mais fournit une limite aux hypothèses que permet d'avancer chaque technique.

4. 3 LES VERRES NATURELS. - Le mélange dans ces verres des deux degrés d'oxydation conduit à mesurer des distances $\mathrm{Fe}-\mathrm{O}$ qui sont la moyenne entre celles mesurées pour un fer (III) tétraédrique et celles mesurées pour un fer (II) octaédrique. On peut remarquer que si l'on tient compte du rapport $\mathrm{Fe}$ (II)/Fe (III) mesuré par effet Mössbauer, on retrouve les distances effectivement mesurées en prenant les valeurs $\mathrm{Fe}$ (II)$\mathrm{O}$ et $\mathrm{Fe}$ (III)- $\mathrm{O}$ du verre d'augite et du verre $\mathrm{Na}_{2} \mathrm{Si}_{2} \mathrm{O}_{5}$ vus plus haut, ce qui montre que l'environnement de ces ions est peu différent.

5. Conclusion. - Les premiers résultats obtenus sur les spectres d'absorption $\mathrm{X}$ dans les verres, dans le cadre d'une étude plus générale sur les éléments $3 \mathrm{~d}$, montrent l'intérêt de cette méthode qui complète les techniques spectroscopiques classiques. Deux types d'informations différents ont été obtenus :

- La position et la structure des seuils confirment que le fer (II) se situe principalement en site octaédrique ;

- L'analyse de l'EXAFS montre que dans le cas $\mathrm{du}$ fer (III), les distances $\mathrm{Fe}-\mathrm{O}$ sont compatibles avec un site tétraédrique, alors que dans le cas du fer (II), les distances mesurées sont plus courtes que celles attendues pour un site hexacoordonné. La distribution radiale des premiers voisins est très faible : ceci est à opposer aux interprétations classiques des élargissements notables des bandes d'absorption sur les spectres optiques, Mössbauer ou R.P.E. [1].

Remerciements. - Nous remercions P. Lagarde et D. Raoux pour le développement de l'expérience EXAFS au L.U.R.E. Le rayonnement synchrotron a été fourni grâce à une coopération avec le Laboratoire de l'Accélérateur Linéaire, et en particulier M. P. Marin. Ce travail est soutenu par la D.G.R.S.T. (A.C.C. «Valorisation des Ressources du Sous-Sol »).

\section{Bibliographie}

[1] Wong, J. et ANgell, C. A., Glass Structure by Spectroscopy, M. Dekker Inc. (New York) 1976.

[2] Raoux, D., Petiau, J., Bondot, P., Calas, G., Fontaine, A., Lagarde, P., Levitz, P., Loupias, G. et Sadoc, A., Revue Phys. Appl. 15 (1980), this volume.

[3] HaYes, J. M., J. non Cryst. Sol. 31 (1978) 57.

[4] Eisenberger, P. et Brown, G. S., Solid State Commun. 29 (1979) 481

[5] Salem, S. J., Chang, C. W., Lee, P. L. et Severson, V., J. Phys. C 11 (1978) 4085.

[6] Kurkjian, C. R. et Sigety, E. A., Phys. Chem. Glasses 9 (1968) 73.
[7] Brown, G. E., Keffer, K. D. et Fenn, P. M., Abstracts Geol. Soc. America, San Diego (1979).

[8] Levitz, P., Thèse Docteur Ingénieur, Paris (1979).

[9] Boon, J. A. et FyFe, W. S., Chem. Geol. 10 (1972) 287.

[10] Levitz, P., Bonnin, D., Calas, G. et Legrand, A. P., J. Phys. $E$. (à paraître).

[11] Edwards, R. J., Paul, A. et Douglas, R. W., Phys. Chem. Glasses 13 (1972) 137.

[12] Mao, H. K., Virgo, B. et Bell P. M., Carnegie Inst. Washington, Yearbook 72 (1973) 631.

[13] FaYe, G. H., Can. Mineral. 11 (1972) 473.

[14] Iwamoto, N., Tsunawaki, Y., Nakagawa, H., Yoshimura, T. et Wakabyashi, I., J. Non Cryst. Sol. 29 (1979) 347. 